\title{
From Gauss graphs to giants
}

\section{Robert de Mello Koch ${ }^{a, b}$ and Lwazi Nkumane ${ }^{b}$}

\author{
${ }^{a}$ School of Physics and Telecommunication Engineering, South China Normal University, \\ Guangzhou 510006, China \\ ${ }^{b}$ National Institute for Theoretical Physics, \\ School of Physics and Mandelstam Institute for Theoretical Physics, \\ University of the Witwatersrand, \\ Wits, 2050, South Africa \\ E-mail: robert@neo.phys.wits.ac.za, lwazi.nkumane@gmail.com
}

ABStract: We identify the operators in $\mathcal{N}=4$ super Yang-Mills theory that correspond to $\frac{1}{8}$-BPS giant gravitons in $\mathrm{AdS}_{5} \times S^{5}$. Our evidence for the identification comes from (1) counting these operators and showing agreement with independent counts of the number of giant graviton states, and (2) by demonstrating a correspondence between correlation functions of the super Yang-Mills operators and overlaps of the giant graviton wave functions.

KEywords: 1/N Expansion, AdS-CFT Correspondence, Brane Dynamics in Gauge Theories

ARXIV EPRINT: 1710.09063 


\section{Contents}

1 Introduction 1

2 Counting 3

$\begin{array}{llr}3 & \text { Matching states to operators } & 6\end{array}$

$\begin{array}{llr}4 \text { Outlook } & 11\end{array}$

\section{Introduction}

The AdS/CFT correspondence [1] provides a beautiful realization of 't Hooft's proposal that the large $N$ limit of Yang-Mills theories are equivalent to string theory [2]. Most studies of the correspondence have focused on the planar limit, which holds classical operator dimensions fixed as we take $N \rightarrow \infty$. There are non-planar large $N$ limits of the theory [3], which are defined by considering operators with a bare dimension that is allowed to scale with $N$ as we take $N \rightarrow \infty$. These limits are relevant for the AdS/CFT correspondence. The limit on which we will focus in this study considers operators with a dimension that scales as $N$. Our focus is on operators relevant for the description of giant graviton branes [4-6].

The worldvolume of the most general $\frac{1}{8}$-BPS giant graviton can be described as the intersection of a holomorphic complex surface in $\mathbb{C}^{3}$ with the five sphere $S^{5}$ of the $\operatorname{AdS}_{5} \times \mathrm{S}^{5}$ spacetime [7]. It is possible to quantize these giant graviton configurations and then to count them [8]. Remarkably, this quantization leads to the Hilbert Space of $N$ noninteracting Bose particles in a $3 \mathrm{~d}$ harmonic oscillator potential, a result conjectured in [9]. In [10] $\frac{1}{8}$-BPS states which carry three independent angular momenta on $S^{5}$ were counted. This counting problem can again be mapped to counting energy eigenstates of a system of $N$ bosons in a 3 -dimensional harmonic oscillator. Both of these analysis $[8,10]$ make use of a world volume description of the branes. Finally, an index to count single trace BPS operators operators has been constructed [11, 12]. The index has been computed both at weak coupling (using the gauge theory) and at strong coupling (as a sum over the spectrum of free massless particles in $\mathrm{AdS}_{5} \times \mathrm{S}^{5}$ ) and the results again agree with $[8,10]$.

Given the AdS/CFT correspondence, this counting should also arise in the dual $\mathcal{N}=4$ super Yang-Mills theory, when the operators of a bare dimension of order $N$ and vanishing anomalous dimension are considered. One of our goals in this study is to demonstrate this.

A crucial ingredient in the study of operators with a bare dimension of order $N$, has been the construction of bases of operators developed in [13-20]. These bases diagonalize the free field theory two point function to all order in $1 / N$ and mix weakly when the Yang-Mills coupling is switched on. Using these bases as a starting point, the spectrum of 
anomalous dimensions for a class operators of bare dimension of order $N$ has been computed in [21-23]. The operators are constructed using the three complex adjoint scalars $Z, Y$ and $X$. We use $n Z \mathrm{~s}, m Y \mathrm{~s}$ and $p X \mathrm{~s}$, fixing $n \sim N$ and $m, p \ll n$. This implies that we are focusing on small deformations of $\frac{1}{2}$-BPS giant gravitons. The operators of a definite scaling dimension are labeled by a permutation $\sigma \in S_{m} \times S_{p}$ and a pair of Young diagrams $R \vdash n+m+p$ and $r \vdash n$. The explicit form of these operators is

$$
\begin{aligned}
O_{R, r}^{\vec{m}, \vec{p}}(\sigma)= & \frac{\left|H_{X} \times H_{Y}\right|}{\sqrt{p ! m !}} \sum_{j, k} \sum_{s \vdash m} \sum_{t \vdash p} \sum_{\vec{\mu}_{1}, \vec{\mu}_{2}} \sqrt{d_{s} d_{t}} \Gamma_{j k}^{(s, t)}(\sigma) \\
& \times B_{j \vec{\mu}_{1}}^{(s, t) \rightarrow 1_{H_{X}} \times H_{Y}} B_{k \vec{\mu}_{2}}^{(s, t) \rightarrow 1_{H_{X}} \times H_{Y}} O_{R,(r, s, t) \vec{\mu}_{1} \vec{\mu}_{2}}
\end{aligned}
$$

The Young diagrams $R$ and $r$ both have $q$ rows for operators dual to a state of $q$ giant gravitons. Each box in $R$ is associated with one of the complex fields, so that we can talk of a box as being a $Z$ box, a $Y$ box or an $X$ box. $r$ collects all of the $Z$ boxes. The difference in the row length of the $q$ th row in $R$ and $q$ th row in $r$ is equal to the number of $X \mathrm{~s}\left(=p_{q}\right)$ and $Y \mathrm{~s}\left(=m_{q}\right)$ in row $q$, so that $R_{q}-r_{q}=m_{q}+p_{q}$. The right most boxes are $X$ boxes, the left most boxes $Z$ boxes and the $Y$ boxes are sandwiched in the middle. The $q$ dimensional vector $\vec{m}$ collects the $m_{i}$, while $\vec{p}$ collects the $p_{i}$. The branching coefficients $B_{j \vec{\mu}}^{(s, t) \rightarrow 1_{H_{Y} \times H_{X}}}$ resolve the operator that projects from $(s, t)$, with $s \vdash m, t \vdash p$, an irreducible representation of $S_{m} \times S_{p}$, to the trivial (identity) representation of the product group $H_{Y} \times H_{X}$ with $H_{Y}=S_{m_{1}} \times S_{m_{2}} \times \cdots S_{m_{q}}$ and $H_{X}=S_{p_{1}} \times S_{p_{2}} \times \cdots S_{p_{q}}$, i.e.

$$
\frac{1}{\left|H_{X} \times H_{Y}\right|} \sum_{\gamma \in H_{X} \times H_{Y}} \Gamma_{i k}^{(s, t)}(\gamma)=\sum_{\vec{\mu}} B_{i \vec{\mu}}^{(s, t) \rightarrow 1_{H_{X} \times H_{Y}}} B_{k \vec{\mu}}^{(s, t) \rightarrow 1_{H_{X} \times H_{Y}}}
$$

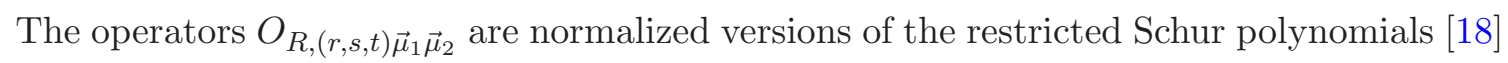

$$
\chi_{R,(r, s, t) \vec{\mu}_{1} \vec{\mu}_{2}}(Z, X, Y)=\frac{1}{n ! m ! p !} \sum_{\sigma \in S_{n+m+p}} \chi_{R,(r, s, t) \vec{\mu}_{1} \vec{\mu}_{2}}(\sigma) \operatorname{Tr}\left(\sigma Z^{\otimes n} Y^{\otimes m} X^{\otimes p}\right),
$$

which themselves provide a basis for the gauge invariant operators of the theory. The restricted characters $\chi_{R,(r, s, t) \vec{\mu}_{1} \vec{\mu}_{2}}(\sigma)$ are defined by tracing the matrix representing group element $\sigma$ in representation $R$ over the subspace giving an irreducible representation $(r, s, t)$ of the subgroup $S_{n} \times S_{m} \times S_{p}$. There is more than one choice for this subspace and the multiplicity labels $\vec{\mu}_{1} \vec{\mu}_{2}$ resolve this ambiguity. The operators $O_{R,(r, s, t) \vec{\mu}_{1} \vec{\mu}_{2}}$ given by

$$
O_{R,(r, s, t) \vec{\mu}_{1} \vec{\mu}_{2}}=\sqrt{\frac{\text { hooks }_{r} \text { hooks }_{s} \text { hookst }_{t}}{\operatorname{hooks}_{R} f_{R}}} \chi_{R,(r, s, t) \vec{\mu}_{1} \vec{\mu}_{2}}
$$

have unit two point function. Although the definition of the Gauss graph operators $O_{R, r}(\sigma)$ is technically rather involved, they have a very natural and simple interpretation in terms of the dual giant graviton branes plus open string excitations. A Gauss graph operator that is labeled by a Young diagram $R$ that has $q$ rows corresponds to a system of $q$ giant gravitons. The $Y$ and $X$ fields describe the open string excitations of the giants. Each such field corresponds to a directed edge, an open string, which can end on any two (not 
necessarily distinct) of the $q$ branes. The permutation $\sigma \in S_{m} \times S_{p}$ is a label which tells us precisely how the $m Y$ 's and the $p X$ 's are draped between the $q$ giant gravitons. The picture of directed edges stretched between $q$ dots is highly suggestive of a brane plus open string system, as reflected in our language. This interpretation is further supported by the fact that the only configurations that appear have the same number of strings starting or terminating on any given giant. This nicely implements the Gauss Law of the brane world volume theory implied by the fact that the giant graviton has a compact world volume. The Gauss graph operators which correspond to BPS states have all open strings described by loops that start at a given giant and loop back to the same giant, i.e. no open strings stretch between giants. In this case, we simply need to specify which brane the open string belongs to and this is most conveniently done by partially labeling Young diagram $R$ : in each box we place a $z$, an $x$ or a $y$. Each row in the operator consists mainly of $Z$ fields, corresponding to the fact that the unexcited giant graviton is dual to a half-BPS operator built only from $Z \mathrm{~s}$. The number of $x$ and $y$ boxes in a given row tell us how many $X$ and $Y$ strings attach to the corresponding giant.

In the next section we will show the counting of these BPS states agrees with the counting of $[8,10]$. Motivated by this observation, we explore the link between the $N$ particle description employing the $3 \mathrm{~d}$ harmonic oscillator and the super Yang-Mills operators in section 3. Our results shed light on the attractive possibility of an $N$ particle description of multi matrix models, suggesting that there maybe an extension of the famous free fermion/eigenvalue description of single matrix models [24]. Finally, we refer the reader to [25] and [26] for further related background dealing with BPS giant gravitons and to [27-29] for further background relevant for the counting and construction of $\frac{1}{4}$ and $\frac{1}{8}$-BPS operators for the regime where operator dimensions are less than $N$.

\section{Counting}

As discussed in the introduction, our description of $\frac{1}{8}$-BPS operators is in terms of a Young diagram $R$ with partially labeled boxes. When the boxes corresponding to $Y$ and $X$ fields are removed from the rows of $R$, we are left with the valid Young diagram $r$. An example of a valid $\frac{1}{8}$-BPS operator is

$$
\begin{array}{|l|l|l|l|l|l|l|l|l|l|l|}
\hline z & z & z & z & z & z & z & z & y & y & x \\
\hline z & z & z & z & z & z & z & y & x & & \\
\cline { 1 - 5 } & z & z & y & y & & \multicolumn{1}{|c|}{} & & \\
\hline
\end{array}
$$

The boxes with label $z$ belong to the Young diagram $r$ and the boxes with label $y$ or $x$ are the ones that are removed from the Young diagram $R$ to obtain $r$. The operator labeled by the Young diagram shown in (2.1) corresponds to a system of 3 giant gravitons, with $2 Y$ strings and an $X$ string attached to the first giant, a $Y$ and an $X$ string attached to the second giant and $2 Y$ strings attached to the third giant. This description in terms of Gauss graph operators is valid in the case where $n$ the total number of boxes of the Young diagram $r$ and $m+p$ the total number of the boxes that are added to the Young diagram $r$ to form $R$, are both large and of order $N \gg 1$. In addition, $m+p \ll n$ and the number 
of rows of the Young diagram $R$ is of order $1=N^{0}$. Finally, the length of any row of $R$ is of order $N$, as is the difference between the length of any two consecutive rows.

Let us first start by fixing our notation. We will denote by $R_{i}$ the number of boxes in the $i$ th row of $R$, and we will denote by $m_{i}$ and $p_{i}$ the number of $Y$ and $X$ boxes to be removed from the $i$ th row of $R$ to obtain $r$. Furthermore, $q$ will stand for the number of rows of $R, n$ will stand for the total number of boxes of $r, m=\sum_{i=1}^{q} m_{i}$ and $p=\sum_{i=1}^{q} p_{i}$. Hence, the total number of boxes of $R$ is then $n+m+p$. If we denote by $r_{i}$ the number of boxes in the $i$ th row of $r$, then we have

$$
r_{i}=R_{i}-m_{i}-p_{i}
$$

In our conventions, we start the numbering of rows from top to bottom. As already mentioned above, this description of $\frac{1}{8}$-BPS states is proved to work [22] in the cases that

$$
R_{i} \sim N \quad R_{i+1}-R_{i} \gg m+p \sim N \quad q \sim N^{0}
$$

We call this the displaced corners approximation because the neighboring corners of $R$ are separated by a huge number of columns. Outside this regime, things are more complicated and it is not even known if partially labeled Young diagrams can be used to describe these $\frac{1}{8}$-BPS states. The number of $\frac{1}{8}$-BPS operators is the same as the number of possible pairs $(R ; r)$ counted with multiplicity equal to the number of ways of assigning a valid vector $\vec{m}=\left(m_{1}, m_{2}, \ldots, m_{q}\right)$. Note that once the pair $(R ; r)$ and the vector $\vec{m}$ are given, the vector $\vec{p}=\left(p_{1}, p_{2}, \ldots, p_{q}\right)$ is determined. The first step towards counting the number of Gauss graph operators entails writing a generating function for the number of pairs $(R ; r)$. Our starting point is the observation that the Young diagrams are in one to one correspondence with partitions of integers. The generating function of the latter is given by

$$
Z=\prod_{n=1}^{\infty} \frac{1}{1-q^{n}}=\sum_{k=0}^{\infty} D_{k} q^{k}
$$

where $D_{k}$ is the number of possible ways to partition an integer $k$. This counting is too coarse for us to reach our goals: we need to track the number of parts in the partition which corresponds to the number of rows in the Young diagram. Indeed, we must encode the information about $q$ the number of rows of $R$, as well as the information about the different possible $m_{i}$ 's and $p_{i}$ 's in such a partition, to ensure that we are counting states in the regime in which the Gauss graph operators provide a trustworthy description. Both modifications are easy to take into account in our case of interest where $m_{i}+p_{i}+r_{i} \ll m_{i+1}+p_{i+1}+r_{i+1}$ for all values of $i=1,2, \ldots, q$. The number of ways to partition an integer $k$ is given by the number of solutions to the equation

$$
k=\sum_{i} \chi_{i} n_{i} \quad n_{1} \geq n_{2} \geq \cdots>0 \quad \chi_{i} \geq 0
$$

Notice that the term $\chi_{i} n_{i}$ in the above equation is associated to the term $\left(q^{n_{i}}\right)^{\chi_{i}}$ in the expansion of $Z$. This term appears in the expansion for the term $\left(1-q^{n_{i}}\right)^{-1}$. Clearly then, to keep track of contributions from different rows $\chi_{i}$ we just need to multiply $q^{n}$ by an 
extra parameter $\chi$ and track the power of $\chi$. So, we consider the following modification of the partition function $Z$

$$
Z=\prod_{i=1}^{\infty} \frac{1}{1-\chi q^{n}}=\sum_{k, d=0}^{\infty} D_{k ; d} \chi^{d} q^{k}
$$

where $D_{k ; d}$ counts the number of Young diagrams with $k$ boxes and $d$ rows. Next consider the information associated to the $m_{i}$ 's and $p_{i}$ 's. There is a potential complication because we want both $R$ and $r$ to be Young diagrams. However, in the displaced corners limit, we can ensure that this is not an issue. Indeed, by taking $m, p \ll\left|r_{i+1}-r_{i}\right|$ for all $i$, we ensure that we can never pile enough $Y$ and $X$ boxes onto a row to make it longer than the row above it. Thus, we may treat the $m_{i}$ 's and $p_{i}$ 's as independent, except for the requirement that $\sum_{i=1}^{q} m_{i}=m$ and $\sum_{i=1}^{q} p_{i}=p$. In terms of the partition function $Z$, this is equivalent to associating to each term $q^{\chi_{i} n_{i}}$, a term $p^{b_{i} m_{i}} r^{c_{i} p_{i}}$, where $b_{i} \neq \chi_{i}$ and $c_{i} \neq \chi_{i}$ in general. The latter condition is equivalent to associating the term $p^{l} r^{m}$, with $l, m=0,1, \ldots$ for each term $q^{n}$ in the product form of $Z$ in equation (2.5). Thus, we finally obtain the generating function

$$
Z=\prod_{l=0}^{\infty} \prod_{m=0}^{\infty} \prod_{n=0}^{\infty} \frac{1}{1-\chi p^{l} r^{m} q^{n}}=\sum_{d, m, p, n} D_{m, p, n ; d} \chi^{d} p^{m} r^{p} q^{n}
$$

where $D_{m, p, n ; d}$ counts the number of diagrams $R$ with $(n+m+p)$ boxes and $d$ rows, that is the result of adding $m+p$ boxes that are randomly distributed over the $d$ rows of the Young diagram $r$ with $n$ boxes. Our construction of the Gauss graph operators only holds when the displaced corners approximation holds. Thus, we trust $D_{m, p, n ; d}$ to count the number of Gauss graph operators for a system of $d \sim N^{0}$ giant gravitons when $n, m, p \sim N$ and $n \gg m+p$. This is the main result of this section.

We want to compare this to the counting of $\frac{1}{8}$-BPS giant gravitons. As we discussed in the introduction, this counting problem can be mapped to counting energy eigenstates of a system of $N$ bosons in a 3 -dimensional harmonic oscillator. The grand canonical partition function for bosons in a 3-dimensional simple harmonic oscillator is given by

$$
Z\left(\zeta, q_{1}, q_{2}, q_{3}\right)=\prod_{n_{1}=0}^{\infty} \prod_{n_{2}=0}^{\infty} \prod_{n_{3}=0}^{\infty} \frac{1}{1-\zeta q_{1}^{n_{1}} q_{2}^{n_{2}} q_{3}^{n_{3}}}
$$

with the fugacity $\zeta$ being dual to particle number [10]. Notice that (2.6) exactly matches the grand canonical partition function (2.7) for bosons in a harmonic oscillator potential with $\chi$ playing the role of the fugacity. This is in harmony with the fact that the number of rows matches the number of giant gravitons. In the 3-dimensional harmonic oscillator we have 3 types of excitations, counted by $q_{1}, q_{2}$ and $q_{3}$. These map into the three types of boxes ( $X, Y$ or $Z$ boxes) appearing in $R$, counted by $p, q$ and $r$. Thus, long rows in $R$ map to highly excited particles. This proves our first claim: the counting of the Gauss graph operators matches the counting of $\frac{1}{8}$ BPS giant gravitons.

It is straightforward to consider the restriction to the $\frac{1}{4}$-BPS giant gravitons. These operators are constructed using only $Z$ and $Y$ fields. Arguing as above and counting partially 
labeled Young diagrams with boxes labeled $z$ or $y$, in the displaced corners approximation, we obtain the generating function

$$
Z=\prod_{l=0}^{\infty} \prod_{n=0}^{\infty} \frac{1}{1-\chi p^{l} q^{n}}=\sum_{d, m, n} D_{m, p, n ; d} \chi^{d} p^{m} q^{n}
$$

This counting can be compared to the counting of $\frac{1}{4}$-BPS giant gravitons. This counting problem can be mapped to counting energy eigenstates of a system of $N$ bosons in a 2-dimensional harmonic oscillator. The counting (2.8) does indeed match the grand canonical partition function for bosons in a 2-dimensional simple harmonic oscillator, which is given by

$$
Z\left(\zeta, q_{1}, q_{2}, q_{3}\right)=\prod_{n_{1}=0}^{\infty} \prod_{n_{2}=0}^{\infty} \frac{1}{1-\zeta q_{1}^{n_{1}} q_{2}^{n_{2}}}
$$

Thus, restricting the counting we demonstrates that the counting of the $\frac{1}{4}$-BPS Gauss graph operators matches the counting of $\frac{1}{4}$ BPS giant gravitons, as it should.

\section{$3 \quad$ Matching states to operators}

The fact that the number of Gauss graph operators matches the number of energy eigenstates states of a system of bosons in a 3-dimensional harmonic oscillator potential, motivates us to look for a correspondence between the two. To start we will consider operators $O_{R, r}^{\vec{m}, \vec{p}}(\sigma)$ labeled by Young diagrams that have a single row. In this case we don't need to encode a complicated shape for $R$, so we will simply list the number of $Z \mathrm{~s}, Y \mathrm{~s}$ and $X \mathrm{~s}$ in the operator as $O_{n, m, p}$. Since this row has $O(N)$ boxes, we have a system of $N$ bosons and one of them is highly excited. The idea is that since we have one highly excited particle, we can use a single particle description and overlaps of the single particle wave functions will match correlation functions of Gauss graph operators in the CFT. We focus on $R$ 's with a single row because the computations are so simple to carry out in this case that we can compute many quantities exactly. There is a simple formula for the Gauss graph operators we consider, in terms of the Schur polynomials

$$
O_{n, m, p}(Z, Y, X)=\mathcal{N} \operatorname{Tr}\left(Y \frac{d}{d Z}\right)^{m} \operatorname{Tr}\left(X \frac{d}{d Z}\right)^{p} \chi_{(n+m+p)}(Z)
$$

where

$$
\mathcal{N}=\sqrt{\frac{n !(N-1) !}{m ! p !(n+m+p) !(N+n+m+p-1) !}}
$$

We are using the notation $(k)$ to denote a Young diagram that has a single row of $k$ boxes. There are a number of natural operators that act on the Gauss graphs. For example, we have

$$
\operatorname{Tr}\left(Y \frac{d}{d Z}\right)^{k_{1}} \operatorname{Tr}\left(X \frac{d}{d Z}\right)^{k_{2}} O_{n, m, p}(Z, Y, X) \propto O_{n-k_{1}-k_{2}, m+k_{1}, p+k_{2}}(Z, Y, X)
$$


Thus, a natural correlator to consider is given by

$$
\left\langle O_{n-k_{1}-k_{2}, m+k_{1}, p+k_{2}}^{\dagger} \operatorname{Tr}\left(Y \frac{d}{d Z}\right)^{k_{1}} \operatorname{Tr}\left(X \frac{d}{d Z}\right)^{k_{2}} O_{n, m, p}\right\rangle=\sqrt{\frac{\left(m+k_{1}\right) !\left(p+k_{2}\right) ! n !}{m ! p !\left(n-k_{1}-k_{2}\right) !}}
$$

To describe a single particle in a $3 \mathrm{~d}$ harmonic oscillator, we need three sets of creation and annihilation operators

$$
\left[a_{z}, a_{z}^{\dagger}\right]=\left[a_{y}, a_{y}^{\dagger}\right]=\left[a_{x}, a_{x}^{\dagger}\right]=1
$$

Using the above oscillators we can create a state with an arbitrary number of $z$ quanta, $y$ quanta or $x$ quanta. We suggest that the correspondence between Gauss graph operators and particle states is as follows

$$
O_{n, m, p} \quad \leftrightarrow \quad\left|O_{n, m, p}\right\rangle=\frac{1}{\sqrt{n ! m ! p !}}\left(a_{x}^{\dagger}\right)^{p}\left(a_{y}^{\dagger}\right)^{m}\left(a_{z}^{\dagger}\right)^{n}|0\rangle
$$

The correspondence identifies the number of $z, y$ or $x$ quanta in the particle state with the number of $Z \mathrm{~s}, Y \mathrm{~s}$ or $X \mathrm{~s}$ in the Gauss graph operator. There is a natural extension to include operators, suggested by this identification. For example

$$
\operatorname{Tr}\left(Y \frac{d}{d Z}\right)^{k_{1}} \operatorname{Tr}\left(X \frac{d}{d Z}\right)^{k_{2}} \quad \leftrightarrow \quad\left(a_{y}^{\dagger}\right)^{k_{1}}\left(a_{x}^{\dagger}\right)^{k_{2}}\left(a_{z}\right)^{k_{1}+k_{2}}
$$

As a test of the proposed correspondence, note that

$$
\begin{aligned}
& \left\langle O_{n-k_{1}-k_{2}, m+k_{1}, p+k_{2}}\left|\left(a_{y}^{\dagger}\right)^{k_{1}}\left(a_{x}^{\dagger}\right)^{k_{2}}\left(a_{z}\right)^{k_{1}+k_{2}}\right| O_{n, m, p}\right\rangle \\
& =\frac{\left\langle 0\left|\left(a_{z}\right)^{n}\left(a_{y}\right)^{m+k_{1}}\left(a_{x}\right)^{p+k_{2}}\left(a_{z}^{\dagger}\right)^{n}\left(a_{y}^{\dagger}\right)^{m+k_{1}}\left(a_{x}^{\dagger}\right)^{p+k_{2}}\right| 0\right\rangle}{\sqrt{n ! m ! p !\left(n-k_{1}-k_{2}\right) !\left(m+k_{1}\right) !\left(p+k_{2}\right) !}} \\
& =\sqrt{\frac{n !\left(m+k_{1}\right) !\left(p+k_{2}\right) !}{\left(n-k_{1}-k_{2}\right) ! m ! p !}}
\end{aligned}
$$

which is in complete agreement with (3.4). Very similar computations comparing, for example

$$
\left\langle O_{n-k_{1}, m-k_{2}, p-k_{3}}^{\dagger}\left(\operatorname{Tr} \frac{d}{d Z}\right)^{k_{1}}\left(\operatorname{Tr} \frac{d}{d Y}\right)^{k_{2}}\left(\operatorname{Tr} \frac{d}{d X}\right)^{k_{3}} O_{n, m, p}\right\rangle
$$

and

$$
\left\langle O_{n-k_{1}, m-k_{2}, p-k_{3}}\left|\left(a_{z}\right)^{k_{1}}\left(a_{y}\right)^{k_{2}}\left(a_{x}\right)^{k_{3}}\right| O_{n, m, p}\right\rangle
$$

show that we should identify

$$
\begin{aligned}
& a_{x} \leftrightarrow \sqrt{\frac{m+n+p}{N+m+n+p}} \operatorname{Tr}\left(\frac{d}{d X}\right) \\
& a_{y} \leftrightarrow \sqrt{\frac{m+n+p}{N+m+n+p}} \operatorname{Tr}\left(\frac{d}{d Y}\right) \\
& a_{z} \leftrightarrow \sqrt{\frac{m+n+p}{N+m+n+p}} \operatorname{Tr}\left(\frac{d}{d Z}\right)
\end{aligned}
$$

These computations make use of the reduction rule of [30, 31]. 
We now want to argue that the identifications we have developed above have a natural extension which identifies Gauss graph operators with $q$ rows with a $q$ particle system. Towards this end, we first point out a dramatic simplification in the formula for the Gauss graph operators, arising when we specialize to BPS operators. As discussed in the introduction, in this case we set the permutation $\sigma$ appearing in (1.1) to the identity. Using the orthogonality of the branching coefficients we then find

$$
\begin{aligned}
\sum_{j, k} \Gamma_{j k}^{(s, t)}(\mathbf{1}) B_{j \vec{\mu}_{1}}^{(s, t) \rightarrow 1_{H_{X} \times H_{Y}}} B_{k \vec{\mu}_{2}}^{(s, t) \rightarrow 1_{H_{X} \times H_{Y}}} & =\sum_{j, k} \delta_{j k} B_{j \vec{\mu}_{1}}^{(s, t) \rightarrow 1_{H_{X} \times H_{Y}}} B_{k \vec{\mu}_{2}}^{(s, t) \rightarrow 1_{H_{X}} \times H_{Y}} \\
& =\delta_{\vec{\mu}_{1} \vec{\mu}_{2}}
\end{aligned}
$$

This leads to the following formula (the operators below are normalized to have a unit two point function; they differ from the operators in (1.1) that are not normalized, by a factor of $\left.\sqrt{\left|H_{X} \times H_{Y}\right|}\right)$

$$
O_{R, r}^{\vec{m}, \vec{p}}(X, Y, Z)=\frac{1}{n ! m ! p !} \sqrt{\frac{\left|H_{X} \times H_{Y}\right| \mathrm{hooks}_{r}}{\operatorname{hooks}_{R} f_{R}}} \sum_{\sigma \in S_{n+m+p}} \operatorname{Tr}\left(P_{R, r} \Gamma_{R}(\sigma)\right) \operatorname{Tr}\left(\sigma X^{\otimes p} Y^{\otimes m} Z^{\otimes n}\right)
$$

$P_{R, r}$ is a projector on the carrier space of $R$. It projects to the subspace of YoungYammonouchi states that have $1,2, \ldots, m+p$ distributed in the boxes that belong to $R$ but not $r$ and $m+p+1, \ldots, m+p+n$ distributed in the boxes that belong to $R$ and $r$. Using this formula, it is straight forward to prove that

$$
\begin{aligned}
& \operatorname{Tr}\left(\frac{d}{d X}\right) O_{R, r}^{\vec{m}, \vec{p}}(X, Y, Z)=\sum_{i=1}^{q} \sqrt{\frac{p_{i} c_{R R_{i}^{(1)}}}{n_{i}+m_{i}+p_{i}}} O_{R_{i}^{(1)}, r}^{\vec{m}, \vec{p}_{i}^{(1)}}(X, Y, Z) \\
& \operatorname{Tr}\left(\frac{d}{d Y}\right) O_{R, r}^{\vec{m}, \vec{p}}(X, Y, Z)=\sum_{i=1}^{q} \sqrt{\frac{m_{i} c_{R R_{i}^{(1)}}}{n_{i}+m_{i}+p_{i}}} O_{R_{i}^{(1)}, r}^{\vec{m}_{i}^{(1)}, \vec{p}}(X, Y, Z) \\
& \operatorname{Tr}\left(\frac{d}{d Z}\right) O_{R, r}^{\vec{m}, \vec{p}}(X, Y, Z)=\sum_{i=1}^{q} \sqrt{\frac{n_{i} c_{R R_{i}^{(1)}}^{(1)}}{n_{i}+m_{i}+p_{i}}} O_{R_{i}^{(1)}, r_{i}^{(1)}}^{\vec{m}, \vec{p}}(X, Y, Z)
\end{aligned}
$$

The first formula above is exact. The last two hold only in the large $N$ limit. We have introduced some new notation: the Young diagram $R_{i}^{(n)}$ is obtained from $R$ by dropping $n$ boxes from row $i$ of $R$. Further, $\vec{p}_{i}^{(n)}$ is obtained from vector $\vec{p}$ by replacing $p_{i} \rightarrow p_{i}-n$ and similarly for $\vec{m}_{i}^{(n)}$. Finally, $c_{R R_{i}^{(1)}}$ is the factor of the box that belongs to $R$ but not to $R_{i}^{(1)}$. Recall that a box in row $i$ and column $j$ has factor $N-i+j$. For the proof of these formulas, we use the notation

$$
\mathcal{N}=\frac{1}{n ! m ! p !} \sqrt{\frac{\left|H_{X} \times H_{Y}\right| \text { hooks }_{r}}{\operatorname{hooks}_{R} f_{R}}}
$$

and

$$
\operatorname{Tr}\left(\sigma \cdot X^{\otimes p} \otimes Y^{\otimes m} \otimes Z^{\otimes n}\right)=X_{i_{\sigma(1)}}^{i_{1}} \cdots X_{i_{\sigma(p)}}^{i_{p}} Y_{i_{\sigma(p+1)}}^{i_{p+1}} \cdots Y_{i_{\sigma(p+m)}}^{i_{p+m}} Z_{i_{\sigma(p+m+1)}}^{i_{p+m+1}} \cdots Z_{i_{\sigma(p+m+n)}}^{i_{p+m+n}}
$$


We will now prove (3.14). A simple computation shows

$$
\begin{aligned}
\frac{d O_{R, r}^{\vec{m}, \vec{p}}}{d X_{i}^{i}} & =p \mathcal{N} \sum_{\sigma \in S_{n+m+p}} \operatorname{Tr}\left(P_{R, r} \Gamma^{(R)}(\sigma)\right) \operatorname{Tr}\left(\sigma \cdot 1 X^{\otimes p-1} \otimes Y^{\otimes m} \otimes Z^{\otimes n}\right) \\
& =p \mathcal{N} \sum_{\sigma \in S_{n+m+p-1}} \sum_{i=1}^{n+m} \operatorname{Tr}\left(P_{R, r} \Gamma^{(R)}(\sigma(i, 1)) \operatorname{Tr}\left(\sigma(i, 1) \cdot 1 X^{\otimes p-1} \otimes Y^{\otimes m} \otimes Z^{\otimes n}\right)\right. \\
& =p \mathcal{N} \sum_{\sigma \in S_{n+m+p-1}} \operatorname{Tr}\left(P_{R, r} \Gamma^{(R)}(\sigma)\left[N+\sum_{i=2}^{n+m}(i, 1)\right]\right) \operatorname{Tr}\left(\sigma \cdot 1 X^{\otimes p-1} \otimes Y^{\otimes m} \otimes Z^{\otimes n}\right)
\end{aligned}
$$

Since we are summing over elements of the subgroup $S_{n+m+p-1} \subset S_{n+m+p}$ we can decompose the trace over the irreducible representation of $S_{n+m+p}$ as a sum of traces over irreducible representation $R_{i}^{(1)}$ of the subgroup $S_{n+m+p-1}$. Now use the fact that $N+$ $\sum_{i=2}^{n+m}(i, 1)$ gives $c_{R R_{i}^{(1)}}=$ the factor of the box dropped from $R$ when acting on any state in the carrier space of $R$ that also belongs to the $R_{i}^{(1)}$ subspace. We find

$$
\frac{d O_{R, r}^{\vec{m}, \vec{p}}}{d X_{j}^{j}}=\sum_{i=1}^{q} f_{\mathcal{N}}^{(i)} c_{R R_{i}^{(1)}} O_{R_{i}^{(1)}, r}^{\vec{m}, \vec{p}_{i}^{(1)}}
$$

where the factor

$$
f_{\mathcal{N}}^{(i)}=\sqrt{\frac{p_{i}}{\left(n_{i}+m_{i}+p_{i}\right) c_{R R_{i}^{(1)}}}}
$$

accounts for the change in the normalization factor $\mathcal{N}$ of the operator. This is an exact formula - it does not depend on large $N$ or on the displaced corners approximation. Next consider the proof of (3.15) and (3.16). Consider

$$
\begin{aligned}
\frac{d O_{R, r}^{\vec{m}, \vec{p}}}{d Y_{i}^{i}} & =m \mathcal{N} \sum_{\sigma \in S_{n+m+p}} \operatorname{Tr}\left(P_{R, r} \Gamma^{(R)}(\sigma)\right) \operatorname{Tr}\left(\sigma \cdot X^{\otimes p} \otimes 1 \otimes Y^{\otimes m-1} \otimes Z^{\otimes n}\right) \\
& =m \mathcal{N} \sum_{\sigma \in S_{n+m+p-1}} \sum_{i=1}^{n+m} \operatorname{Tr}\left(P_{R, r} \Gamma^{(R)}(\sigma) \operatorname{Tr}\left((p+1,1) \sigma(p+1,1) \cdot 1 \otimes X^{\otimes p} \otimes Y^{\otimes m-1} \otimes Z^{\otimes n}\right)\right. \\
& =m \mathcal{N} \sum_{\sigma \in S_{n+m+p-1}} \operatorname{Tr}\left(P_{R, r} \Gamma^{(R)}((1, p+1) \sigma)(1, p+1)\right) \operatorname{Tr}\left(\sigma \cdot 1 \otimes X^{\otimes p} \otimes Y^{\otimes m-1} \otimes Z^{\otimes n}\right)
\end{aligned}
$$

The new feature in the above derivation is the presence of the $(1, p+1) \in S_{n+m+p}$ factors needed to swap the removed $Y$ box to the end of the row so that it can be removed, using the same manipulations as above. The evaluation of the action of these factors is most easily performed using Young's orthogonal representation, which gives a rule for the action of adjacent permutations (i.e. permutations of the form $(i, i+1)$ ) on Young-Yamanouchi (hereafter abbreviated YY) states. Let $|Y\rangle$ denote a YY state, and let $|Y(i \leftrightarrow i+1)\rangle$ denote the YY state obtained by swapping boxes $i$ and $i+1$. A box in row $a$ and column 
$b$ has content given by $b-a$. Denote the content of the box in $|Y\rangle$ filled with $j$ by $c_{j}$. The rule is

$$
(i, i+1)|Y\rangle=\frac{1}{c_{i}-c_{i+1}}|Y\rangle+\sqrt{1-\frac{1}{\left(c_{i}-c_{i+1}\right)^{2}}}|Y(i \leftrightarrow i+1)\rangle
$$

This rule simplifies dramatically in the displaced corners limit, at large $N$. If the two boxes belong to the same row we find $(i, i+1)|Y\rangle=|Y\rangle$ and if not $(i, i+1)|Y\rangle=|Y(i \leftrightarrow i+1)\rangle$. This is all that is needed to complete the proof of (3.15) and (3.16) and it proceeds exactly as for the first rule proved above. Note that because we used simplifications of the large $N$ limit, (3.15) and (3.16) are not exact statements but hold only at large $N$. The three statements derived above admit some natural generalizations. For example, we can consider tracing over a product of derivatives to obtain

$$
\operatorname{Tr}\left(\frac{d^{k}}{d X^{k}}\right) O_{R, r}^{\vec{m} \vec{p}}(X, Y, Z)=\sum_{i=1}^{q}\left(\frac{c_{R R_{i}^{(1)}}}{n_{i}+m_{i}+p_{i}}\right)^{\frac{k}{2}} \prod_{a=0}^{k-1} \sqrt{p_{i}-a} O_{R_{i}^{(k)}, r}^{\vec{p}_{i}^{(k)}} \vec{m}(X, Y, Z)
$$

There are obvious generalization when we have a product of $Y$ or $Z$ derivatives. We could also allow more than one type of derivative in a given trace, for example (in what follows $\left.k=k_{1}+k_{2}\right)$

$$
\begin{aligned}
& \operatorname{Tr}\left(\frac{d^{k_{1}}}{d X^{k_{1}}} \frac{d^{k_{2}}}{d Y^{k_{2}}}\right) O_{R, r}^{\vec{m} \vec{p}}(X, Y, Z) \\
& =\sum_{i=1}^{q}\left(\frac{c_{R R_{i}^{(1)}}}{n_{i}+m_{i}+p_{i}}\right)^{\frac{k}{2}} \prod_{a=0}^{k_{1}-1} \sqrt{p_{i}-a} \prod_{b=0}^{k_{2}-1} \sqrt{m_{i}-b} O_{R_{i}^{(k)}, r}^{\vec{m}_{i}^{\left(k_{2}\right)} \vec{p}_{i}^{\left(k_{1}\right)}}(X, Y, Z)
\end{aligned}
$$

By using these formulas for each trace successively, we can also easily evaluate expressions of this form

$$
\operatorname{Tr}\left(\frac{d^{k_{1}}}{d X^{k_{1}}} \frac{d^{k_{2}}}{d Y^{k_{2}}}\right) \cdots \operatorname{Tr}\left(\frac{d^{k_{3}}}{d Z^{k_{3}}}\right) O_{R, r}^{\vec{m} \vec{p}}(X, Y, Z)
$$

To compare to a multi particle system of $q$ noninteracting particles, again in a 3 -dimensional harmonic oscillator potential, we need to introduce $q$ copies of the oscillators $(I, J=$ $1, \ldots, q)$

$$
\left[a_{z}^{(I)}, a_{z}^{(J) \dagger}\right]=\left[a_{y}^{(I)}, a_{y}^{(J) \dagger}\right]=\left[a_{x}^{(I)}, a_{x}^{(J) \dagger}\right]=\delta^{I J}
$$

one copy for each particle. Each Gauss graph operator $O_{R, r}^{\vec{m} \vec{p}}$ is specified by giving the number of $Z$ boxes $\left(r_{i}\right), Y$ boxes $\left(m_{i}\right)$ and $X$ boxes $\left(p_{i}\right)$ in the $i$ th row for $i=1, \ldots, q$. The corresponding multi particle state is

$$
\left|O_{R, r}^{\vec{m} \vec{p}}\right\rangle=\prod_{I=1}^{q} \frac{\left(a_{z}^{(I) \dagger}\right)^{r_{I}}}{\sqrt{r_{I} !}} \frac{\left(a_{y}^{(I) \dagger}\right)^{m_{I}}}{\sqrt{m_{I} !}} \frac{\left(a_{x}^{(I) \dagger}\right)^{p_{I}}}{\sqrt{p_{I} !}}|0\rangle
$$

Using these formulas we can compare (for example) the matrix elements

$$
\left\langle O_{R_{q}^{(k)}, r_{q}^{(k)}}^{\vec{m} \vec{p}}\left|\left(a_{z}^{(I)}\right)^{k}\right| O_{R, r}^{\vec{m} \vec{p}}\right\rangle
$$


to the correlation functions

$$
\left\langle O_{R_{q}^{(k)}, r_{q}^{(k)}}^{\vec{m} \vec{\dagger}} \operatorname{Tr}\left(\frac{d^{k}}{d Z^{k}}\right) O_{R, r}^{\vec{m} \vec{p}}\right\rangle
$$

to learn that we should identify

$$
\operatorname{Tr}\left(\frac{d^{k}}{d Z^{k}}\right) \leftrightarrow \sum_{I=1}^{q}\left(\sqrt{\frac{N+m_{I}+n_{I}+p_{I}}{m_{I}+n_{I}+p_{I}}}\right)^{k}\left(a_{z}^{(I)}\right)^{k}
$$

In the above formula $n_{I}$ is the number of $Z$ boxes in row $I, m_{I}$ the number of $Y$ boxes and $p_{I}$ the number of $X$ boxes. The general rule is $\left(k=k_{1}+k_{2}+k_{3}\right)$

$$
\operatorname{Tr}\left(\frac{d^{k_{1}}}{d X^{k_{1}}} \frac{d^{k_{2}}}{d Y^{k_{2}}} \frac{d^{k_{3}}}{d Z^{k_{3}}}\right) \leftrightarrow \sum_{I=1}^{q}\left(\sqrt{\frac{N+m_{I}+n_{I}+p_{I}}{m_{I}+n_{I}+p_{I}}}\right)^{k}\left(a_{x}^{(I)}\right)^{k_{1}}\left(a_{y}^{(I)}\right)^{k_{2}}\left(a_{z}^{(I)}\right)^{k_{3}}
$$

It is easy to check that the ordering of operators inside the trace on the left hand side above does not matter, when acting on the operators we consider, at large $N$. Multi trace formulas use the above identification for each trace separately. For example

$$
\begin{aligned}
\operatorname{Tr} & \left(\frac{d^{k_{1}}}{d X^{k_{1}}} \frac{d^{k_{2}}}{d Y^{k_{2}}} \frac{d^{k_{3}}}{d Z^{k_{3}}}\right) \operatorname{Tr}\left(\frac{d^{k_{4}}}{d X^{k_{4}}}\right) \\
\leftrightarrow & \sum_{I=1}^{q}\left(\sqrt{\frac{N+m_{I}+n_{I}+p_{I}}{m_{I}+n_{I}+p_{I}}}\right)^{k}\left(a_{x}^{(I)}\right)^{k_{1}}\left(a_{y}^{(I)}\right)^{k_{2}}\left(a_{z}^{(I)}\right)^{k_{3}} \\
& \times \sum_{J=1}^{q}\left(\sqrt{\frac{N+m_{J}+n_{J}+p_{J}}{m_{J}+n_{J}+p_{J}}}\right)^{k_{4}}\left(a_{x}^{(I)}\right)^{k_{4}}
\end{aligned}
$$

By comparing overlaps between states with polynomials of creation and annihilation operators sandwiched in between and correlators of Gauss graph operators with traces of polynomials of the matrices and derivatives with respect to the matrices acting on the Gauss graph operators as in the examples we studied above, we can build any entry in the dictionary between the $q$ particle system and Gauss graph operators with $q$ rows.

\section{Outlook}

The description of giant gravitons, constructed using a world volume analysis, allows one to count the set of all $\frac{1}{8}$-BPS giant gravitons. This counting matches $N$ bosons in a 3 dimensional harmonic oscillator. It is also possible to define an index to count single trace BPS operators, and it can be computed both at weak coupling (using the gauge theory) and at strong coupling (as a sum over the spectrum of free massless particles in $\operatorname{AdS}_{5} \times \mathrm{S}^{5}$ ). The results of these different computations are in complete accord. One can compute the spectrum of anomalous dimensions, for operators with a bare dimension of order $N$, in the $\mathcal{N}=4$ super Yang-Mills theory [21-23]. In this study we have demonstrated that exactly the same counting (i.e. $N$ bosons in a 3 -dimensional harmonic oscillator) results from 
counting operators of vanishing anomalous dimension in this spectrum. Motivated by this agreement, we have looked for a relation between multi particle wave functions and Gauss graph operators. Our basic result is that a map between particle wave functions for particles in a 3-dimensional harmonic oscillator and Gauss graph operators is easily constructed by comparing overlaps of wave functions of the particle system with correlators of Gauss graph operators. The correlator computations have made use of significant simplifications that arise for the BPS Gauss graph operators. The number of particles match the number of rows in the Young diagram labeling the Gauss graph operator. In our opinion, these results provide concrete evidence that the Gauss graph operators are indeed the operators dual to the $\frac{1}{8}$-BPS giant gravitons. To interpret the link between the particle system and the Gauss graph operators, recall the link between giant gravitons and an eigenvalue description of the multi matrix dynamics, which has been pursued in $[32,33]$. Thus, the fact that the matrix model computations appear to be related to the dynamics of noninteracting particles gives hints as to how matrix model dynamics may simplify, along the line of the proposals of [34-37].

Any computation of overlaps performed with our wave functions can be mapped into a computation of Gauss graph correlators. However, the wave function picture does clarify the structure of the $\frac{1}{8}$-BPS operators in ways that are not manifest in the Gauss graph description. For example, our wave functions make it clear that a Hilbert space for $N 3 \mathrm{~d}$ bosons emerges from the CFT. This structure is interesting, as we now explain. The $\frac{1}{2}$-BPS sector has a high degree of supersymmetry and so is relatively simple and has often served as a bridge connecting the gauge theory and supergravity regimes. In the CFT this sector can be consistently decoupled resulting in a system that admits a description in terms of free fermions moving in a harmonic oscillator potential. This is well understood from the gauge theory point of view where the Lagrangian of the decoupled theory is that of a complex matrix whose eigenvalues obey Fermi-Dirac statistics, with the statistics induced from the integration measure. On the gravity side the symplectic form of Type IIB SUGRA encodes the commutation relations that must be imposed to quantize the system. Restricting this symplectic form to the LLM family of solutions defines a symplectic structure that fixes a quantization and ultimately reproduces the free fermion Hilbert space [38]. In the same way that free fermion quantum mechanics is equivalent to the singlet sector of a single matrix quantum mechanics, the wave functions we have written down are equivalent to the BPS Gauss graph operators. It would be interesting to recover the Hilbert space of our wave functions by quantizing using the symplectic form of Type IIB SUGRA, after restricting to the $\frac{1}{8}$-BPS family of solutions.

\section{Acknowledgments}

RdMK would like to thank Ilies Messamah and Sanjaye Ramgoolam for many useful discussions on the topic of this study. This work is based upon research supported by the South African Research Chairs Initiative of the Department of Science and Technology and National Research Foundation. Any opinion, findings and conclusions or recommendations expressed in this material are those of the authors and therefore the NRF and DST do not accept any liability with regard thereto. 
Open Access. This article is distributed under the terms of the Creative Commons Attribution License (CC-BY 4.0), which permits any use, distribution and reproduction in any medium, provided the original author(s) and source are credited.

\section{References}

[1] J.M. Maldacena, The large- $N$ limit of superconformal field theories and supergravity, Int. J. Theor. Phys. 38 (1999) 1113 [hep-th/9711200] [INSPIRE].

[2] G. 't Hooft, A Planar Diagram Theory for Strong Interactions, Nucl. Phys. B 72 (1974) 461 [INSPIRE].

[3] V. Balasubramanian, M. Berkooz, A. Naqvi and M.J. Strassler, Giant gravitons in conformal field theory, JHEP 04 (2002) 034 [hep-th/0107119] [INSPIRE].

[4] J. McGreevy, L. Susskind and N. Toumbas, Invasion of the giant gravitons from Anti-de Sitter space, JHEP 06 (2000) 008 [hep-th/0003075] [INSPIRE].

[5] A. Hashimoto, S. Hirano and N. Itzhaki, Large branes in AdS and their field theory dual, JHEP 08 (2000) 051 [hep-th/0008016] [INSPIRE].

[6] M.T. Grisaru, R.C. Myers and O. Tafjord, SUSY and goliath, JHEP 08 (2000) 040 [hep-th/0008015] [INSPIRE].

[7] A. Mikhailov, Giant gravitons from holomorphic surfaces, JHEP 11 (2000) 027 [hep-th/0010206] [INSPIRE].

[8] I. Biswas, D. Gaiotto, S. Lahiri and S. Minwalla, Supersymmetric states of $N=4$ Yang-Mills from giant gravitons, JHEP 12 (2007) 006 [hep-th/0606087] [INSPIRE].

[9] C.E. Beasley, BPS branes from baryons, JHEP 11 (2002) 015 [hep-th/0207125] [INSPIRE].

[10] G. Mandal and N.V. Suryanarayana, Counting 1/8-BPS dual-giants, JHEP 03 (2007) 031 [hep-th/0606088] [INSPIRE].

[11] J. Kinney, J.M. Maldacena, S. Minwalla and S. Raju, An Index for 4 dimensional super conformal theories, Commun. Math. Phys. 275 (2007) 209 [hep-th/0510251] [INSPIRE].

[12] C. Romelsberger, Counting chiral primaries in $N=1, D=4$ superconformal field theories, Nucl. Phys. B 747 (2006) 329 [hep-th/0510060] [InSPIRE].

[13] S. Corley, A. Jevicki and S. Ramgoolam, Exact correlators of giant gravitons from dual $N=4$ SYM theory, Adv. Theor. Math. Phys. 5 (2002) 809 [hep-th/0111222] [INSPIRE].

[14] V. Balasubramanian, D. Berenstein, B. Feng and M.-x. Huang, D-branes in Yang-Mills theory and emergent gauge symmetry, JHEP 03 (2005) 006 [hep-th/0411205] [INSPIRE].

[15] T.W. Brown, P.J. Heslop and S. Ramgoolam, Diagonal multi-matrix correlators and BPS operators in $N=4$ SYM, JHEP 02 (2008) 030 [arXiv:0711.0176] [INSPIRE].

[16] Y. Kimura and S. Ramgoolam, Branes, anti-branes and brauer algebras in gauge-gravity duality, JHEP 11 (2007) 078 [arXiv: 0709. 2158] [INSPIRE].

[17] Y. Kimura and S. Ramgoolam, Enhanced symmetries of gauge theory and resolving the spectrum of local operators, Phys. Rev. D 78 (2008) 126003 [arXiv:0807.3696] [INSPIRE].

[18] R. Bhattacharyya, S. Collins and R. de Mello Koch, Exact Multi-Matrix Correlators, JHEP 03 (2008) 044 [arXiv: 0801.2061] [INSPIRE]. 
[19] R. Bhattacharyya, R. de Mello Koch and M. Stephanou, Exact Multi-Restricted Schur Polynomial Correlators, JHEP 06 (2008) 101 [arXiv:0805.3025] [INSPIRE].

[20] T.W. Brown, P.J. Heslop and S. Ramgoolam, Diagonal free field matrix correlators, global symmetries and giant gravitons, JHEP 04 (2009) 089 [arXiv:0806.1911] [INSPIRE].

[21] W. Carlson, R. de Mello Koch and H. Lin, Nonplanar Integrability, JHEP 03 (2011) 105 [arXiv: 1101.5404] [INSPIRE].

[22] R. de Mello Koch, M. Dessein, D. Giataganas and C. Mathwin, Giant Graviton Oscillators, JHEP 10 (2011) 009 [arXiv:1108.2761] [InSPIRE].

[23] R. de Mello Koch and S. Ramgoolam, A double coset ansatz for integrability in AdS/CFT, JHEP 06 (2012) 083 [arXiv:1204.2153] [INSPIRE].

[24] E. Brézin, C. Itzykson, G. Parisi and J.B. Zuber, Planar Diagrams, Commun. Math. Phys. 59 (1978) 35 [inSPIRE].

[25] J. Pasukonis and S. Ramgoolam, Quantum states to brane geometries via fuzzy moduli spaces of giant gravitons, JHEP 04 (2012) 077 [arXiv: 1201.5588] [INSPIRE].

[26] M.C. Abbott, J. Murugan, A. Prinsloo and N. Rughoonauth, Meromorphic Functions and the Topology of Giant Gravitons, Phys. Lett. B 730 (2014) 215 [arXiv:1312.4900] [INSPIRE].

[27] M. Bianchi, J.F. Morales and H. Samtleben, On stringy $A d S_{5} \times S^{5}$ and higher spin holography, JHEP 07 (2003) 062 [hep-th/0305052] [INSPIRE].

[28] T.W. Brown, Cut-and-join operators and $N=4$ super Yang-Mills, JHEP 05 (2010) 058 [arXiv: 1002.2099] [INSPIRE].

[29] J. Pasukonis and S. Ramgoolam, From counting to construction of BPS states in $N=4$ SYM, JHEP 02 (2011) 078 [arXiv: 1010.1683] [INSPIRE].

[30] R. de Mello Koch and R. Gwyn, Giant graviton correlators from dual $\mathrm{SU}(N)$ super Yang-Mills theory, JHEP 11 (2004) 081 [hep-th/0410236] [INSPIRE].

[31] R. de Mello Koch, J. Smolic and M. Smolic, Giant Gravitons - with Strings Attached (I), JHEP 06 (2007) 074 [hep-th/0701066] [INSPIRE].

[32] D. Berenstein, A toy model for the AdS/CFT correspondence, JHEP 07 (2004) 018 [hep-th/0403110] [INSPIRE].

[33] D. Berenstein, Giant gravitons: a collective coordinate approach, Phys. Rev. D 87 (2013) 126009 [arXiv:1301.3519] [INSPIRE].

[34] D. Berenstein, Large-N BPS states and emergent quantum gravity, JHEP 01 (2006) 125 [hep-th/0507203] [INSPIRE].

[35] R. de Mello Koch, Geometries from Young diagrams, JHEP 11 (2008) 061 [arXiv:0806.0685] [INSPIRE].

[36] D. Berenstein, Sketches of emergent geometry in the gauge/gravity duality, Fortsch. Phys. 62 (2014) 776 [arXiv:1404.7052] [INSPIRE].

[37] R. de Mello Koch, D. Gossman, L. Nkumane and L. Tribelhorn, Eigenvalue Dynamics for Multimatrix Models, Phys. Rev. D 96 (2017) 026011 [arXiv: 1608.00399] [INSPIRE].

[38] L. Grant, L. Maoz, J. Marsano, K. Papadodimas and V.S. Rychkov, Minisuperspace quantization of 'Bubbling AdS' and free fermion droplets, JHEP 08 (2005) 025 [hep-th/0505079] [INSPIRE]. 